\title{
Competencias profesionales del docente de educación desde la perspectiva de profesores y estudiantes de una universidad privada en Lima, Perú
}

\author{
Sandra Natalia Mazuelos Bravo \\ smazuelos@gmail.com \\ https://orcid.org/0000-0002-1252-6283 \\ Escuela de Posgrado \\ Universidad Nacional Mayor de San Marcos \\ Lima, Perú
}

\section{RESUMEN}

Se presentan los resultados de una investigación para optar el grado de Doctor en Educación. El estudio se planteó los objetivos de identificar las competencias profesionales del docente de la facultad de educación de una universidad privada de Lima, Perú, desde la percepción de sus estudiantes y profesores; comprender el sistema de las competencias profesionales y las dimensiones implicadas y; presentar una propuesta de dimensiones competenciales y definiciones conceptuales que identificaran las competencias profesionales del profesor de dicha facultad. La investigación eligió un abordaje metodológico cualitativo (estudio de caso). Se examinaron puntos de vista y valoraciones de profesores y estudiantes, recogidos mediante grupos focales y entrevistas semi-estructuradas. Los datos se organizaron en base a categorías identificadas a priori y se analizaron mediante el Método Comparativo Constante. El análisis permitió tratar categorías emergentes, refinar, integrar, reducir y absorber categorías e ir construyendo modelos teóricos iniciales, intermedios y finales (o teorización). La teorización realizada, sugiere que profesores y estudiantes identifican dos dimensiones competenciales, dentro de las competencias profesionales de los docentes de la facultad: 1) las competencias personales y 2) las laborales. Estas a la vez, están conformadas por grupos de subcompetencias las cuales son discutidas más adelante.

Palabras Clave: competencias profesionales; estudio de caso; Método Comparativo Constante; competencias personales; competencias laborales 


\title{
Professional skills of education professors from the perspective of professors and students of a private university in Lima, Peru
}

\begin{abstract}
The results of an investigation to opt for the degree of Doctor of Education are presented. The study set out the objectives of identifying the professional skills of the professor of the faculty of education of a private university in Lima, Peru, from the perception of their students and professors; understand the system of professional competencies and the dimensions involved and; present a proposal of competence dimensions and conceptual definitions that identify the professional competences of the professor of said faculty. The research chose a qualitative methodological approach (case study). The views and assessments of professors and students, collected through focus groups and semistructured interviews, were examined. The data was organized based on categories identified a priori and analyzed using the Constant Comparative Method. The analysis allowed treating emerging categories, refining, integrating, reducing and absorbing categories and building initial, intermediate and final theoretical models (or theorizing). The theorizing carried out suggests that professors and students identify two competency dimensions within the professional competencies of education professors: 1) personal competencies and 2) labor competencies. These, at the same time, are made up of groups of sub-competencies which are discussed later.
\end{abstract}

Key Words: professional competencies; case study; Constant Comparative Method; personal skills; labor competencies

Artículo recibido: 30 noviembre. 2021 Aceptado para publicación: 29 diciembre 2021

Correspondencia: smazuelos@gmail.com Conflictos de Interés: Ninguna que declarar 


\section{INTRODUCCIÓN}

La evolución social e histórica genera cambios epistemológicos que cuestionan la manera en la que se genera y valida el conocimiento. El actual paradigma de la educaciónformación parece ya no contener las respuestas a las necesidades del mundo, por ello, requiere ser cambiado. Un punto de partida es la identificación de competencias profesionales del rol docente que permitan confrontar los parámetros teóricos, con las necesidades específicas de estudiantes, de instituciones educativas y las del contexto con las prácticas educativas que, además, acorten la brecha entre lo que la educación necesita y lo que ofrece.

La génesis del término competencia, vinculado a los procesos productivos generados por el cambio tecnológico, aparece en el artículo llamado Testing for Competence Rather Than for "Intelligence" de David McClelland, en 1973. Este sugería que, en los procesos de reclutamiento de personal, la evaluación de competencias era más relevante como predictor de idoneidad para un puesto, que las evaluaciones psicométricas y de inteligencia comúnmente usadas en la época. Afirmaba que parecía más sabio abandonar la búsqueda de factores de habilidad pura y seleccionar pruebas que fueran válidas de manera que las puntuaciones cambiasen a medida que la persona crece en experiencia, sabiduría y habilidad para desempeñarse efectivamente en varias tareas que la vida le presenta. Más adelante, la competencia se empieza a describir como un "Conjunto de características de una persona que están relacionadas directamente con una buena ejecución en una determinada tarea o puesto de trabajo" (Boyatzis, 1982).

En la actualidad se reconoce el papel decisivo que tienen las personas en el desarrollo y éxito de las de organizaciones Dolan, Valle, Jackson, \& Schuler, (2007), gracias al aporte que hacen a través de sus habilidades, destrezas y actitudes. Por ello, es fundamental identificar el perfil específico de competencias profesionales de cada profesión y las necesidades particulares de cada organización. Esto no es distinto dentro de la organización educativa. Pues esto permite que cada individuo potencie los logros institucionales, a la vez, que incrementa sus propias potencialidades.

El formador de formadores, hoy, no solo tiene el desafío de lograr estudiantes académicamente exitosos, sino también de colaborar en la formación de ciudadanos activos, críticos y comprometidos. Esto requiere alto nivel técnico y académico (competencias técnicas o laborales), sensibilidad y permeabilidad a los problemas que 
nacen de las transformaciones culturales, sociales y económicas actuales. Además, capacidad de gestionar sosteniblemente, la incertidumbre y las emociones generadas por dichos cambios (competencias blandas o personales). De aquí la necesidad de una selección de docentes basadas en competencias profesionales que, según López Arce, impulsen la formación y educación hacia un mejor equilibrio entre las necesidades de los individuos, las organizaciones y la sociedad en general (2006).

Estudios realizados en Etiopía encontraron que existe una relación significativa entre el desarrollo de las competencias docentes y el rendimiento académico de los estudiantes (Ugbe \& Agim, 2009, Marinković, Bjekić, \& Zlatić, 2012). Mientras que los estudios de McKinsey Corporation (2007) refuerzan la centralidad del profesor de alta calidad en las escuelas con mayor desempeño del mundo. Estos resultados coinciden con un reporte de la OECD de 2005 (citado por Donaldson, 2012), que afirma que la calidad de los docentes (docente de alto rendimiento, en contraste con uno de bajo rendimiento) es uno de los factores que más conduce a mejoras sustanciales en el aprendizaje y el éxito escolar. Asimismo, Donaldson (2012), sostiene que los maestros son el ingrediente más importante en la escuela cuando se trata del rendimiento de los estudiantes y que la calidad de un sistema educativo se basa en la calidad de sus maestros.

Existen investigaciones sobre el tema de competencias profesionales docentes que preceden el estudio sobre el que reporta este artículo. Así, la investigación "Estrategias didácticas para el desarrollo de competencias básicas del profesional universitario peruano" (Natalia Rodríguez, 2011). En las conclusiones identifica elementos competenciales de carácter técnico (vinculados a la didáctica) y elementos de carácter personal (vinculados a los valores).

Alex Pavié (2012) presentó la tesis doctoral "Las Competencias Profesionales del Profesorado de Lengua Castellana y Comunicaciones en Chile: Aportaciones a la Formación Inicial (Universidad de Valladolid). Pavié afirma que Formación Inicial docente debe cambiar y fortalecerse

y que estos cambios incluyen ámbitos que van desde la actualización de los conocimientos de parte de los formadores de formadores, pasando por que el estudiante (futuro profesor) tenga contacto con las aulas lo más pronto posible y que tenga una apropiación conceptual afín a lo que demandan los paradigmas de aprendizaje que sustentan los diseños curriculares basados en competencias. En el caso de Chile, es el paradigma de aprendizaje 
constructivista. (capítulo tercero, p.414)

Así también Pavié encontró que existe una mayor valoración del dominio del saber en las diferentes competencias profesionales que se reconocen como tales. Por otro lado, encontró que "no existe un modelo o diseño curricular basado en competencias unificado que gestione e integre en forma eficiente estos tres dominios el saber (...); el saber hacer (...) y saber ser (...) en la práctica laboral y/o profesional”. (p.422).

Gonzalo Rivera (2006), en la tesis doctoral: "Modelo de competencias profesionales para docentes de educación básica según el criterio de un grupo de educadores", afirma "que las instituciones formadoras de docentes imparten un currículo disociado del ejercicio profesional" (p.165). Otra interesante conclusión a la que se arribó es "que los planes de estudios desde preescolar hasta universidad se proyectan en base a competencias profesionales; y la formación que se recibe en las instituciones formadoras de docentes, está ajena a esta metodología” (p.166).

De acuerdo con análisis realizado en "Analysis on Literature Review of Competency" (Su-Chin Hsieh, Jui-Shin Lin, Hung-Chun Lee, 2012), existen diversas perspectivas para clasificar las competencias: desde la capacidad conceptual, el comportamiento y los conocimientos/habilidades. De acuerdo al Modelo Iceberg aplicado para el análisis, los conocimientos y habilidades son fácilmente identificables y visibles y aparecen en la parte superior del iceberg. Estos fueron desarrollados y mejorados a través de la educación y la capacitación para el trabajo con relativa facilidad. Por otro lado, los motivos y rasgos aparecen en la base del iceberg, son más propensos a ser escondidos pues comprenden la parte más interna de la personalidad de un individuo. Por lo tanto, son más difíciles de desarrollar y reformar a través de la educación escolar y la capacitación.

\section{ESTRATEGIAS METODOLÓGICAS, MATERIALES Y MÉTODOS}

En el caso estudiado, nos planteamos identificar las dimensiones competenciales, implicadas en las competencias profesionales del docente de educación, y las subcompetencias que las conforman, desde la perspectiva de estudiantes y docentes de la Facultad de Educación de una universidad privada de Lima. De igual forma, se buscaba elaborar una propuesta de dimensiones competenciales y definiciones conceptuales, en base a los hallazgos realizados durante la investigación.

En la etapa preparatoria de la investigación, sub-etapa de reflexión y diseño, se elaboró un supuesto de trabajo que proporcionó los ejes temáticos para la búsqueda revisión 
bibliográfica de literatura especializada. El supuesto afirma que los docentes de la facultad de educación, cuentan con competencias profesionales docentes, de carácter personal (blandas) y laboral (duras), que aportan al capital humano de la institución y coadyuvan a una mejor formación del futuro profesor de educación. Al respecto por Garrido, C. (2007) aporta argumentos teóricos que señalan que el capital humano es definido como el conocimiento que los individuos adquieren durante su vida y que usan para producir buenos servicios o ideas en el mercado o fuera de él.

Se identificaron dos categorías generales o macro categorías, de manera apriorística, surgida del tema de investigación y de la investigación bibliográfica (Ver Figura 1):

1. "Competencias personales" $\mathrm{y}$

2. "Competencias laborales"

Estas generaron, diez sub-categorías para hacer la indagación más detallada.

Figura 1 Categorías Iniciales

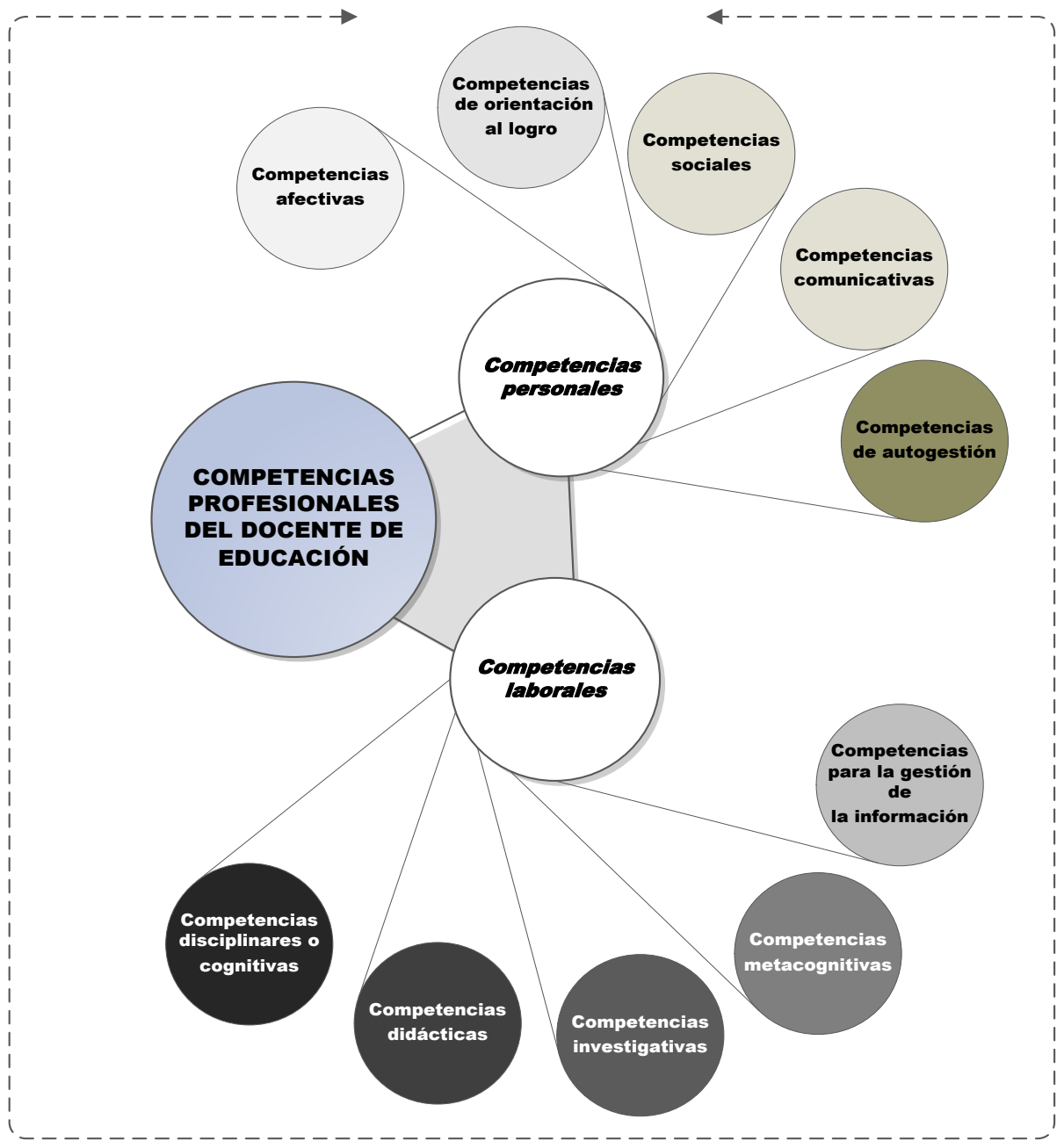

Mazuelos, S. (2016) 
Estas categorías y sub-categorías se consideraron como punto de partida, pues a lo largo del acopio de datos, algunas categorías desaparecieron y otras emergieron.

\subsection{Definición Nominal Conceptual de Categorías y Subcategorías de Análisis}

Se definió cada una de las dos categorías identificadas a priori y sub-categorías, de la manera cómo se esperaba encontrarlas en la realidad de caso estudiado

\section{Tabla 1}

Definición Nominal Conceptual de Categorías y Subcategorías de Análisis

\section{COMPETENCIAS PERSONALES (Humanas o blandas):}

Habilidades sociales, conductas y disposiciones de tipo actitudinal y afectivas, adquiridas por experiencia propia, que complementan el uso del conocimiento técnico.

- Competencias afectivas: Son medidas a través del saber-hacer flexible en el contexto familiar, social, cultural y laboral, permiten actuar en contextos complejos y móviles, globalizados más que regionales y con relaciones de incertidumbre y requieren de habilidades intrapersonales e interpersonales del individuo (Parra, 2005).

- Competencias de orientación al logro: Vinculadas al buen desempeño y al logro de resultados. Implican el esfuerzo que hacen las personas como individuos y el equipo de trabajo como unidad, por cumplir con los objetivos planteados en un proyecto, dentro de las expectativas esperadas (Spencer, L \& Spencer, S.,1993), con responsabilidad, organización, capacidad para alinear los objetivos personales con los organizacionales. A fin de medir esta competencia se puede tomar como referencia la superación personal en relación con el rendimiento pasado; la orientación a resultados basado en medida objetiva, de competitividad en correspondencia con el desempeño y superación de los demás; el mejoramiento continuo en concordancia con resultados que uno mismo se ha propuesto, de innovación esperando realizar algo único y excepcional (Estrada, s.f.) 
- Competencias sociales: Hacen posible la identificación de las propias conductas y las condiciones conductuales de las otras personas. Facilitan el trabajo cooperativo, en equipo, y las acciones de liderazgo (Tejada, 2009 y Parra s.f.).

- Competencias comunicativas: La competencia comunicativa del profesional es la potencialidad que tiene el sujeto de lograr una adecuada interacción comunicativa a partir del dominio e integración en el ejercicio profesional de los conocimientos acerca del proceso de comunicación humana, habilidades comunicativas, principios, valores, actitudes y voluntad para desempeñarse en su profesión eficientemente así como para tomar decisiones oportunas ante situaciones complejas o nuevas, que faciliten el logro de los objetivos trazados o propuestos en diferentes contextos y en las dimensiones afectivocognitiva, comunicativa y sociocultural (Aguirre,, 2005). "[...]Es considerada una competencia potenciadora de otras que suponen el cumplimiento de objetivos tanto interpersonales como organizacionales”. Barraycoa y Lasaga, Universitat Abat Oliba CEU (s.f. p.5).

- Competencias de autogestión: Hacen posible la gestión de uno mismo a través del manejo efectivo del tiempo; el establecimiento de objetivos; prioridades y estándares; el asumir la responsabilidad del propio aprendizaje; el escuchar activamente y con interés; el empleo de una variedad de habilidades académicas (análisis, síntesis, argumentación); el desarrollar y adaptar estrategias de aprendizaje; el demostrar flexibilidad mental; y el empleo del aprendizaje en distintas situaciones; el planificar y trabajar en torno a objetivos de medio y largo alcance; la reflexión sobre el propio aprendizaje; clarificar con espíritu crítico pero de forma constructiva; y el manejo del estrés. (Bennet et al. 1999, citado por García, M.B., 2011).

\section{COMPETENCIAS LABORALES:}

Conocimientos técnicos o especializados: Constituyen una plataforma de conductas con una fuerte base científica, industrial, mecánica y tecnológica requeridas para ser exitoso en el trabajo y se desarrollan a través de la educación, la capacitación o durante el servicio. Se orientan a resultados predecibles basados en procedimientos y metodologías.

- Competencias para la gestión de la información: Permiten emplear recursos de información apropiados; usar pertinentemente la tecnología; emplear los recursos adecuadamente; manejar volúmenes significativos de información con efectividad, 
emplear un lenguaje y formas apropiados a una variedad de actividades; interpretar formas de información variada; presentar ideas e información; responder a diferentes propósitos, audiencias y contextos; emplear la información de forma crítica; y emplear la información en forma innovadora y creadora (Bennet, Dunne y Carré , 1999, citado por García, M.B. , 2011).

- Competencias meta-cognitivas: Permiten la reflexión y autocrítica sobre la propia práctica con el propósito de mejorarla. (Tejada, 2009)

- Competencias investigativas: Permiten el dominio de los métodos científicos, el diagnóstico de problemas pedagógicos, la elaboración de proyectos de investigación y la valoración e impacto de la actividad científica Orama tamine y Varcárcel (2013).

- Competencias didácticas: Se centran en el uso consciente de conocimientos, capacidades, habilidades y destrezas para provocar el aprendizaje. Permiten al docente, identificar y transformar el saber de referencia, los contenidos y la información, en saber aprendido por sus alumnos y en la posibilidad de seguir aprendiendo (Junta de Castilla y León, s.f.). Involucradas en la dirección del proceso de enseñanza-aprendizaje, la planificación de estrategias de aprendizaje, la elaboración y gestión de medios de enseñanza y el diseño del sistema de evaluación (Orama, et al 2013).

\section{- Competencias disciplinares o cognitivas (Conocimiento académico):}

Relacionadas con un "conjunto de concepciones disciplinares, relativas al currículo o a las ciencias de la educación, saberes que se generan en el proceso de formación inicial. Son explícitos y están organizados, atendiendo a la lógica disciplinar, en el mejor de los casos" (Demuth, 2011, p. 34). Permiten que las actividades dentro de una ocupación sean realizadas con un estándar definido y sostenible en el tiempo.

Mazuelos (2016)

\subsection{Diseño}

Se realizó la investigación desde una perspectiva y un enfoque cualitativo basado en un estudio de caso centrado en "las competencias profesionales del docente de la Facultad de Educación de una universidad privada de Lima (Perú) desde la perspectiva de los estudiantes de segundo semestre y los docentes de educación de dicha facultad. 
El diseño permitió hacer uso de: la visión holística y global del fenómeno; además, un trato detallado e intenso del mismo (Regehr, Stern, \& Shlonsky, 2007, p.411, citados por Eunjung, Mishna \& Brennenstuhl, 2010) y posibilitó estar en contacto con la realidad para capturar las perspectivas de los protagonistas y el contexto del fenómeno. Igualmente, el carácter de provisionalidad de algunas decisiones, hizo posible ir adaptando la investigación según fueron emergiendo nuevas necesidades (Ruíz, 2003).

La investigación se desarrolló desde un nivel descriptivo. La recolección de datos se hizo a partir muestras de estudiantes y profesores extraídas por Muestreo Teórico con el propósito de generar teoría hasta la saturación de las categorías (saturación de datos o sin información nueva).

El análisis de la información fue realizado mediante el Método Comparativo Constante. Su herramienta principal de análisis, la contrastación sistemática de dos o más fenómenos (los primeros datos y regularidades de las verbalizaciones), permitió sacar conclusiones, conceptos o teorías que a la vez eran contrastados y corregidos sobre la base de nueva evidencia. Así nuevos términos, conceptos y modelos teóricos (iniciales, intermedios y finales) fueron emergiendo hasta concluir el análisis hasta la saturación de los datos.

La recolección de datos se hizo mediante la técnica del grupo focal o entrevista con una guía de quince preguntas semiestructuradas para docentes y estudiante, por separado. La guía fue sometida al juicio de expertos, quienes sugirieron mejoras. Estas fueron incorporadas, antes de la prueba piloto, que, a la vez, originó nuevos ajustes.

Las fases, etapas y actividades del proceso de investigación, se establecieron en base a Rodríguez, Gil y García (2005) cuya estructura para la investigación cualitativa facilitó la organización del estudio de caso a nivel de etapas; mientras que la estructura más detallada de Bolseguí, M. y Fuguet A, (2006) posibilitó la identificación de etapas, subetapas y actividades. Estas se detallan en la Tabla 2. 
Tabla 2 Fases, etapas y actividades del proceso de investigación

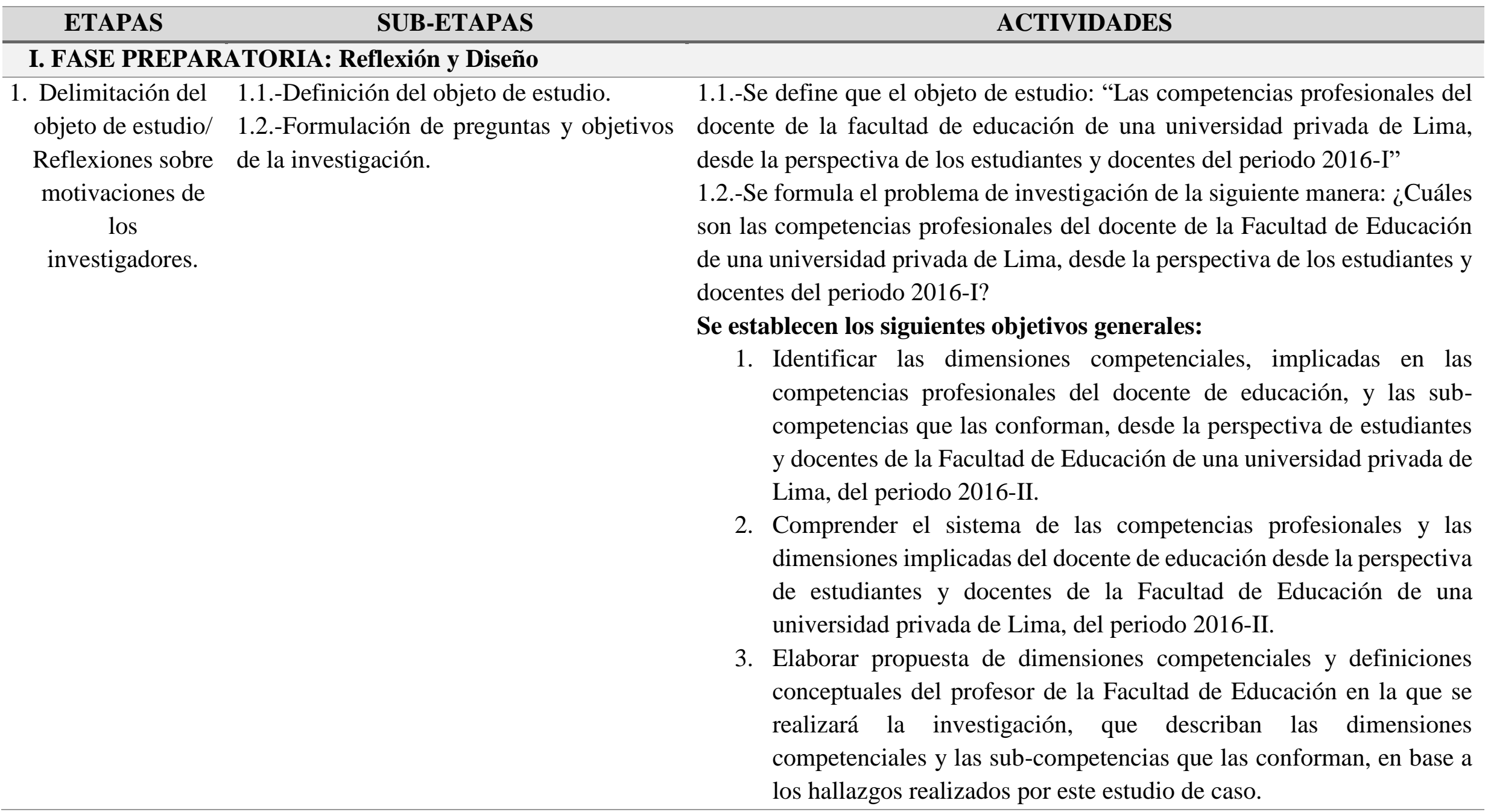




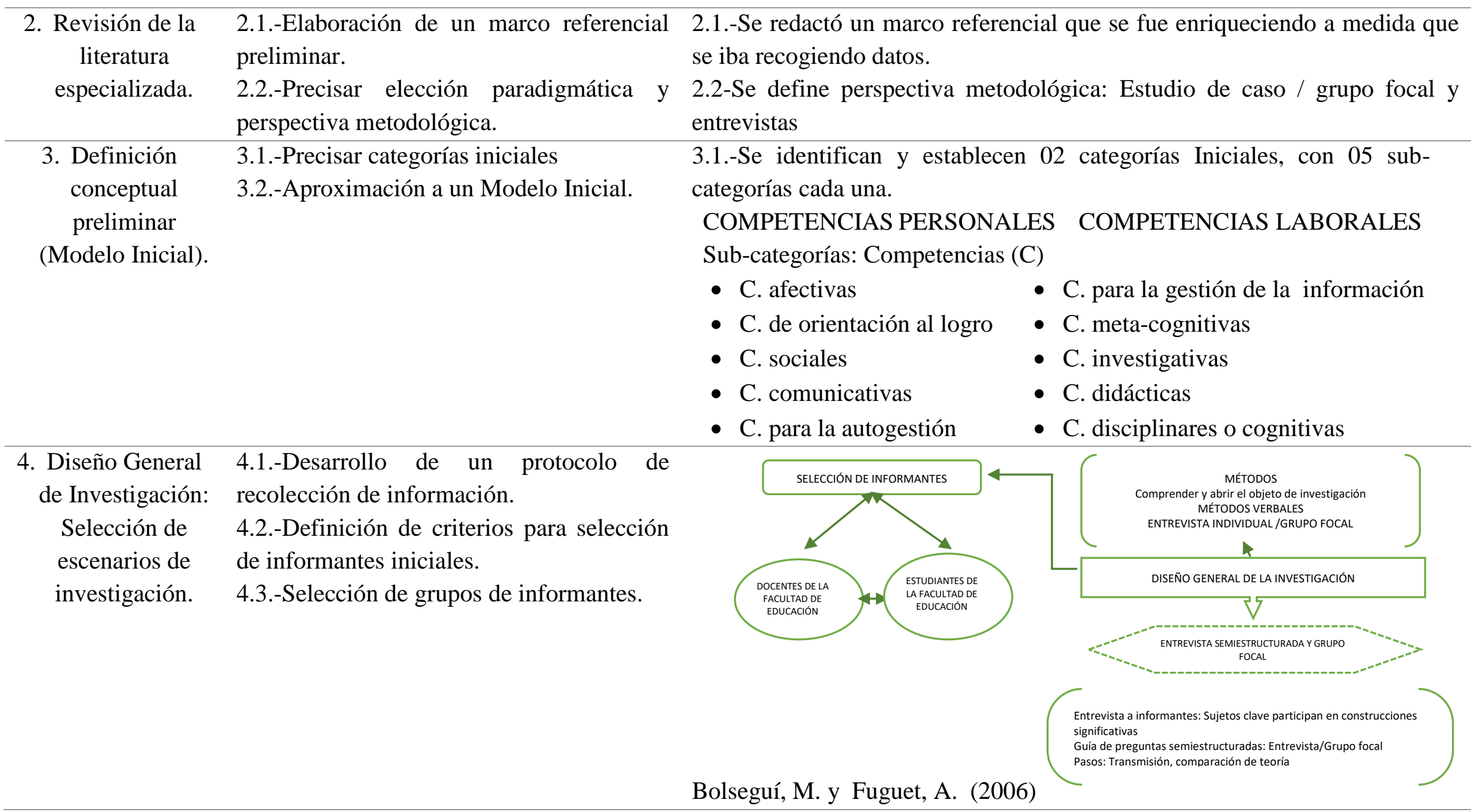




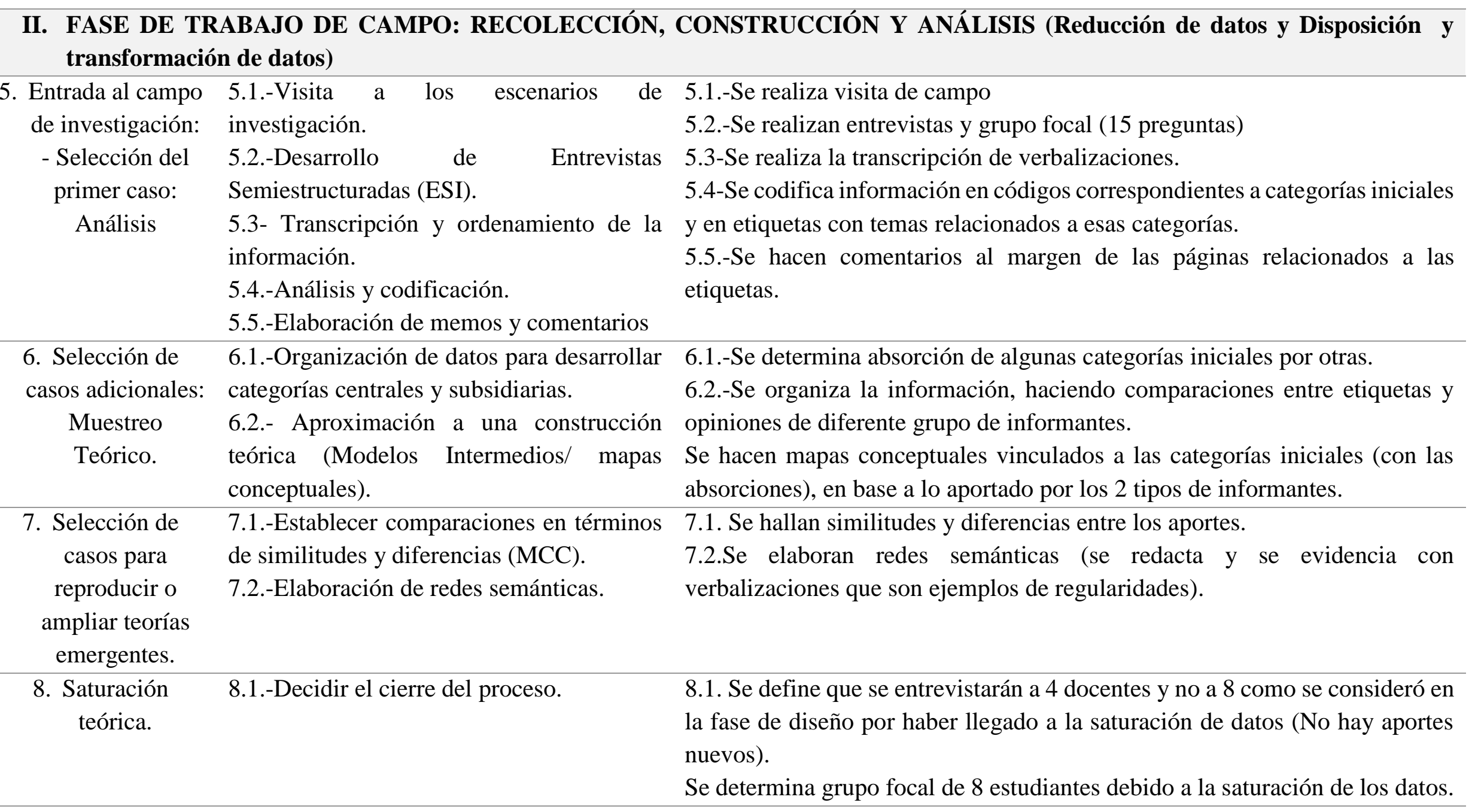




\section{FASE DE TEORIZACIÓN}

9. Delimitar y 9.1.-Comparar teorías emergentes con la redactar la teoría: literatura especializada en términos de Integración de un similitudes y diferencias (MCC).

Modelo Final 9.2.-Triangulación de datos y teorías.

9.1.-9.2.-Se elabora tabla comparativa (triangulación) entre lo que los informantes dicen sobre determinada categoría, lo que dice la literatura revisada y lo que dice la investigadora.
9.3.-Integración de ideas (Elaboración de
un Modelo Final).

\section{FASE DE ELABORACIÓN DEL INFORME DE INVESTIGACIÓN}

-Se elaboró el informe del caso investigado.

Fuente: Elaboración Mazuelos (2016), en base a Bolseguí, M. y Fuguet A, (2006) y Rodríguez, Gil y García (2005) 


\section{RESULTADOS Y DISCUSIÓN}

Para el proceso validación de la investigación cualitativa seguimos Maxell (1992), representante del modelo paradigmático de validez (citado por Sandín, M.P. p.226), quien señala que la comprensión es un concepto mucho más importante que el de validez que, aunque se relacionan con la validez de los textos y no con los datos o métodos, son necesarias para añadir confiabilidad a la investigación y complementar el método de comparativo constante. De los cinco tipos de validez que presenta Maxwell relacionadas con las formas de comprensión inherentes a la investigación cualitativa: 1) Validez descriptiva, 2) Validez interpretativa, 3) validez teórica, 4) Generalización interna y 5) Generalización externa. Para este proceso de la investigación se tomaron los primeros tres tipos de validez de los textos.

\subsection{Resultados}

De acuerdo a la teorización realizada, se confirmó que, desde la perspectiva de los informantes, las competencias profesionales del profesor de la facultad de educación tienen dos dimensiones competenciales: Por un lado, está la dimensión de Competencias Personales, conformada por dos sub-competencias: las competencias afectivas y sociales (las competencias comunicativas aparecen inmersas dentro de estas últimas). Por otro lado, está la dimensión de Competencias Laborales, que se presenta más compleja al estar conformada por cuatro sub-competencias: 1) Las competencias de orientación al logro. 2) Las competencias disciplinares o cognitivas, dentro de las que se encuentran las competencias investigativas, 3) Las competencias didácticas, dentro de las que se encuentran las competencias metodológicas y 4) Las competencias para la gestión de la información, cuyo único ángulo identificado es las competencias para el uso de TIC para la enseñanza y el aprendizaje (Ver Figura $\mathrm{N}^{\circ} 2$ ). Los resultados obtenidos por esta investigación se circunscriben al contexto del caso estudiado. 
Figura 2 Las competencias profesionales del docente de la facultad de educación

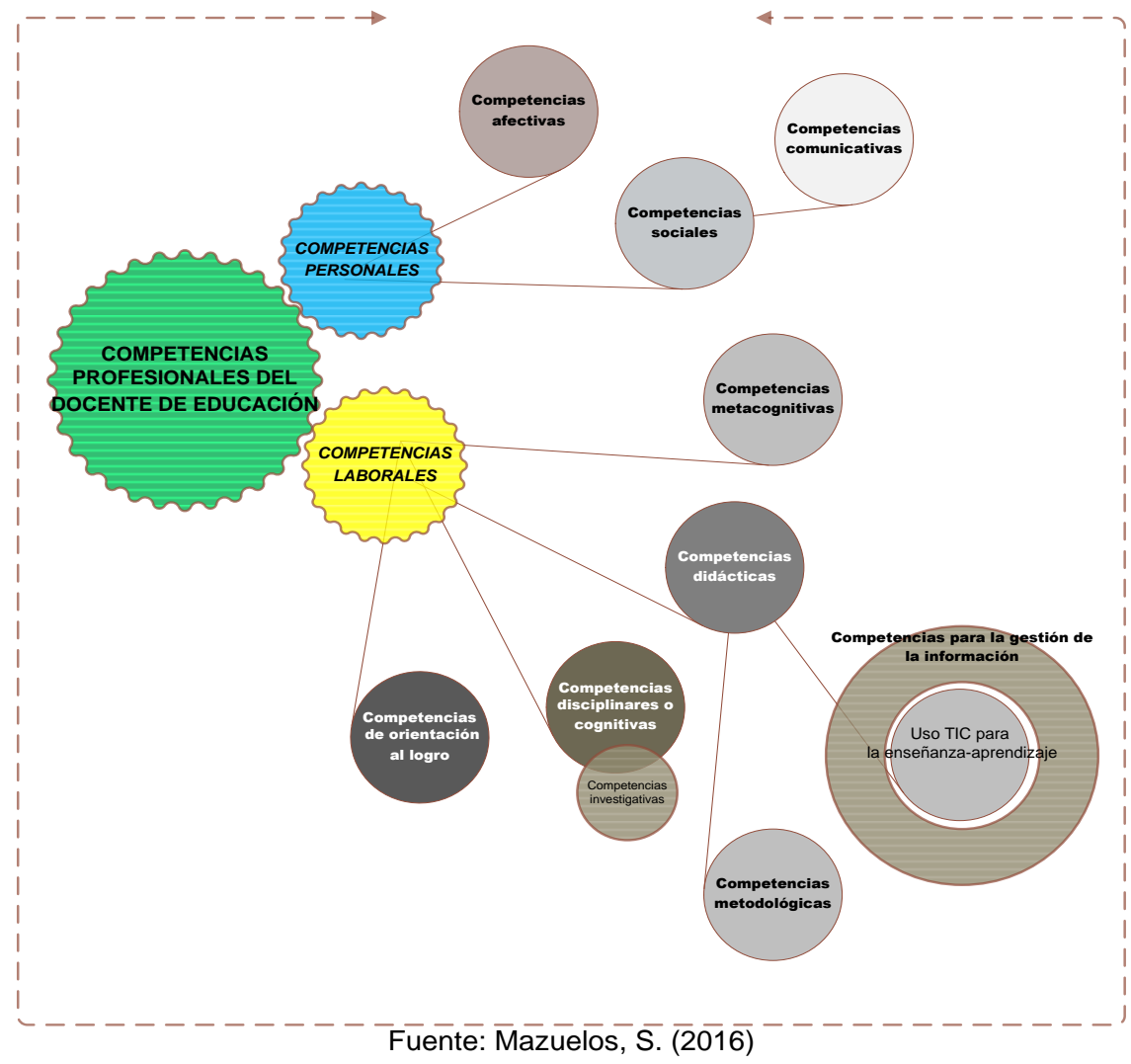

\subsection{Discusión}

El Método Comparativo Constante hizo posible la elaboración de teorías iniciales e intermedias y finales (Ver Tabla 2, p.11). De este proceso de análisis cabe destacar la etapa de triangulación de teorías entre fuentes y referentes (1. Los modelos teóricos elaborados a partir de regularidades en las verbalizaciones del grupo de estudiantes y de docentes entrevistados; 2. Las teorías de los referentes teóricos y; 3. la postura de la investigadora) ya que los puntos de vista comparados, no necesariamente convergieron en una misma dirección, sino que presentaban un panorama de cómo está el estado del arte en el aspecto tratado. Por esta razón, se tomó solo aquello que se alineaba con el problema, el supuesto de trabajo y los objetivos de la investigación. A partir de la integración de ideas, se elaboraron conceptos que pasaron a conformar un modelo teórico final. En la Tabla 3 se presentan los modelos iniciales y finales obtenidos durante el proceso de contrastación.

Los modelos finales sirvieron de insumo para construir la propuesta de dimensiones competenciales y definiciones conceptuales que identifican las competencias profesionales del profesor de la facultad en la que se realizó el estudio de caso. 


\section{Tabla 3}

Modelos iniciales y modelos finales

\begin{tabular}{|c|c|c|c|}
\hline Categoría & ncias & & $\begin{array}{l}\text { Modelo } \\
\text { (Integra }\end{array}$ \\
\hline $\begin{array}{lr}\text { la facultad en } & \text { su } \\
\text { proceso } & \text { de } \\
\text { establecer } & \\
\text { relaciones } & \\
\text { cercanas, } \\
\text { humanas } \\
\text { empáticas con los } \\
\text { estudiantes. Le } \\
\text { permiten al } \\
\text { docente entender } \\
\text { la problemática } \\
\text { del estudiante y } \\
\text { atender sus }\end{array}$ & $\begin{array}{l}\text { Competencia } \\
\text { s sociales }\end{array}$ & $\begin{array}{l}\text { El docente de educación } \\
\text { establece relaciones cercanas al } \\
\text { sintonizarse intelectual y } \\
\text { emocionalmente con el } \\
\text { estudiante para su bienestar } \\
\text { integral. De esta manera, crea } \\
\text { un buen clima en la comunidad } \\
\text { de la facultad y favorece el } \\
\text { ámbito académico y el trabajo } \\
\text { en equipo. }\end{array}$ & $\begin{array}{l}\text { Contar con competencias sociales implica que el docente debe desarrollar la capacidad } \\
\text { para la comunicación efectiva (en los lenguajes académicos, científicos, y de } \\
\text { comunicación expresiva y receptiva), respeto, actitudes pro sociales, asertividad, etc. } \\
\text { (Bisquerra, s.p). De igual forma, el docente debe ser una persona capaz de interactuar } \\
\text { promoviendo la integración y convivencia en el respeto, democracia e igualdad de } \\
\text { derechos, y un conductor cultura (Perrenoud, 2001). } \\
\text { Las competencias sociales hacen posible que el docente pueda sintonizarse intelectual y } \\
\text { emocionalmente con el estudiante y establecer relaciones positivas para su bienestar } \\
\text { integral. Así también debe mantener relaciones positivas con sus colegas, de manera que } \\
\text { se facilite un ambiente académico y de trabajo en equipo. Las relaciones cercanas con los } \\
\text { estudiantes, construidas a partir de las competencias sociales permiten también hacer al } \\
\text { docente más permeable a las peculiaridades y necesidades de su estudiante, prevenir } \\
\text { situaciones y solucionar sus problemas a través de acciones de liderazgo, de cooperación } \\
\text { y de persuasión (Pérez, 2005 y Ayala, 2008, citados por Tejada, 2009). }\end{array}$ \\
\hline
\end{tabular}


necesidades $\quad \mathrm{y}$

expectativas.

Para el docente de educación, la comunicación y la capacidad de escucha son herramientas con

Competencias las que establece relaciones con comunicativas otros para lograr los objetivos de aprendizaje así como, resolver los problemas de los estudiantes.

\section{COMPETENCIA}

\section{S LABORALES}

Se relacionan con

el conjunto de

conocimientos

formales, técnicos

o especializados

que necesita e

profesor para

desempeñar

exitosamente las

actividades de su

función docente.

Estas

competencias

están

estrechamente

vinculadas a los

grupos

competenciales

disciplinares

El logro del docente de

educación es la formación

Competencias integral del estudiante como un

de orientación ser humano reflexivo que

al logro

entiende las individualidades y alcanza desempeños medibles.

El docente de educación usa tecnologías de la Información y la comunicación (TIC) de manera limitada debido a la

Competencias brecha en el uso de TIC entre para la gestión profesores y estudiantes; a la de la falta de manejo de la didáctica información: del uso de TIC; el temor a Uso de TIC perder el control de los estudiantes y a la necesidad de evidencia física del avance. Esto resulta en la falta de innovación en el uso TIC,
Las competencias comunicativas, van más allá del manejo de los lenguajes formales, académicos o didácticos: Involucran, además, expresarse física y verbalmente con coherencia y asertividad, es escuchar con atención activa y apertura.

Estas competencias son de vital importancia para el docente de educación pues le permiten lograr relaciones positivas con el estudiante; facilitar todo el proceso de aprendizaje, lograr los objetivos así como, resolver los problemas de los estudiantes.

Las competencias de orientación al logro del docente de educación están vinculadas a lo que logra a nivel profesional. Según esto, la atención y el trabajo docente deben estar enfocados a lograr un ser humano reflexivo, que entiende las individualidades, que ejercen una ciudadanía global, es decir que es alguien que valora la diversidad y la interconectividad, que es solidario, y que respeta los derechos de los otros y que, además, es un profesional competente. Esto ciertamente se logra con una educación diseñada para lograr un equilibrio entre las demandas de la propia formación profesional y las de la sociedad (López Arce, 2006), a través de desempeños medibles.

Dentro las sub-Competencias para la gestión de la información, se encuentra el uso de TIC. El docente de hoy requiere no solo saber cómo gestionar la información adecuada, en la forma más pertinente y ser capaz de recuperarla apropiadamente. Requiere, sobremanera, manejar el uso de las tecnologías de la información y la comunicación con propósitos de enseñanza-aprendizaje, dentro de la dinámica de avance del contexto histórico-social (Galvis, R., 2007). Esto es para que el estudiante no solamente se beneficie de las mejores prácticas docentes en el aula, sino también pueda recibir la orientación y ayuda para el desarrollo de competencias informática y digitales que ha adquirido empíricamente.

Esta necesidad implica que el docente de educación supere la brecha en el uso de TIC entre profesores y estudiantes. Requiere también que tenga un dominio de la didáctica para el uso de TIC. Que comprenda la funcionalidad, los beneficios, el proceso creativo del conocimiento a través de las TIC, a fin de no ceder al temor a perder el control de los 


\section{cognitivos,}

investigativos,

didácticos y meta

cognitivos. disminución de la motivación y

en la necesidad que el profesor de la facultad desarrolle habilidades para el uso de TIC, con propósitos de enseñanzaaprendizaje.

El docente de educación debe tener conocimientos relativos a su profesión en teoría y práctica y más allá del currículo, Competencias disciplinares o cognitivas

\section{conocimientos generales,}

conocimientos sobre la realidad

y ser sensible a la diversidad, además debe conocer de pedagogía, de investigación vinculada a la realidad para ser capaz de gestionar la innovación y tener experticias. estudiantes y a la necesidad de evidencia física del avance. Este manejo le permitirá una mayor innovación en el uso TIC, para mantener la motivación en alto y lograr formar futuros docentes van aprendiendo y adaptándose a los cambios tecnológicos que se vayan presentando para que sean capaces de ir innovando con el propósito de ayudar a sus estudiantes a beneficiarse del uso eficiente de las tecnologías de la información y la comunicación.

El docente de educación precisa no solo conocer su área disciplinar, en los aspectos teóricos y prácticos, requiere también conocimientos sobre la realidad, con un amplio horizonte cultural y que sea sensible a la diversidad para que pueda intervenir de manera comprometida como ciudadano del mundo (Galvis, 2007).

De igual manera, debido al contexto cambiante actual, se necesita de un docente capaz de gestionar la incertidumbre (Bar, 1999, citado por Galvis, 2007). Por ello, es vital que sepa de pedagogía que le facilite investigar, diseñar adecuadamente para la situación y momento en que los que le ha tocado desempeñarse y tomar decisiones convenientes para formar estudiantes con las competencias precisas para atender las necesidades de la sociedad en la que está inserto.

- $\quad$ Competencias investigativas

Las competencias investigativas aparecen absorbidas dentro de los saberes que el docente de educación debe tener. Estas aparecen vinculadas a la necesidad de que el docente promueva y motive la investigación; aparece con condicionantes: La investigación que realiza el docente debe estar relacionada con la realidad, debe servir para identificar los mejores contenidos para el beneficio de los estudiantes; debe servir para ser aplicada a nivel currículo y a nivel de la didáctica en el aula; y sobre todo debe ser la base de la innovación. 
El docente de educación debe reflexionar sobre lo que se quiere para a educación y la Competencias práctica educativa, teniendo meta- como centro al estudiante para cognitivas tener objetivos claros. Debe estar motivado y tener compromiso para la autoevaluación y la mejora.
Las sub-competencias meta-cognitivas son "la búsqueda de oportunidades más allá de los recursos disponibles, cualidad que tienen algunas personas junto con la habilidad para evaluar en forma simultánea, el éxito y el fracaso" (Correa, 2009, p.77).

Desde esta perspectiva, actuar por la inercia de la corriente educativa, actuar por impulsividad o de manera irreflexiva no tiene cabida cuando se forman seres humanos pues puede acarrear graves consecuencias.

El docente de educación debe desarrollar la meta cognición como una forma de razonar y reflexionar, a diferentes niveles, sobre sus propias concepciones sobre la educación y sobre las mejores prácticas en el aula para formar profesionales y ciudadanos globales competentes. Implica analizar, aprender, desaprender, aplicar para mejorar de forma continua. La meta cognición por lo general es un proceso individual, por ello, el docente debe llevar un paso más allá este proceso de reflexión, pues es necesario que los docentes vayan encontrando consenso en lo que se quiere para la educación según el contexto social, del país, global, en el cómo se beneficia mejor al estudiante, o en lo que requiere el estudiante particular en la situación particular en la que se labora. La mejora personal que se encuentra a través de los procesos meta cognitivos son importantes insumos, pero requieren ser articulados para lograr resultados. Se necesitan entonces, metas claras, motivación, compromiso para la autoevaluación y trabajo consensuado para la mejora.

El docente de educación, Dentro de las competencias laborales, las sub-competencias didácticas el docente de aunque sabe llegar al estudiante, debe definir la meta de la didáctica de la facultad. Si bien, adapta su metodología, Competencias que usa a la comunicación didácticas

como base para cubrir necesidades particulares, necesita incorporar el desarrollo de competencias a la didáctica, planificando con flexibilidad educación deben definir las metas que persigue. Esto demanda tener competencias de planificación, competencias para la selección y secuencialización de los contenidos didáctica, competencias de evaluación de procesos entre otras; deben también considerar las expectativas que tiene el estudiante

Y dentro de las competencias didácticas, las competencias metodológicas, aquellas implican crear condiciones para despertar u orientar la curiosidad intelectual, establecer conexiones entre las diversas experiencias, formar hábitos, actitudes e intereses subyacentes y permanentes hacia el aprendizaje; interrelacionar la curiosidad orgánica de exploración física (tocar, manipular, palpar) y la interrogación lingüística (por qué, para qué, cómo). (Barrón, M. 2009). 
para atender las expectativas de los estudiantes. De igual forma, requiere adoptar una metodología dinámica y paciente que incluya no solamente el desarrollo de competencias, el uso de TIC, sino también fases prácticas que involucre salir del campus y lo lleve al logro de las metas.
Una vez que el objetivo de aprendizaje haya quedado explicitado, el docente de educación debe ser capaz de diseñar una metodología que responda a los logros que se esperan para una formación integral, adecuada al contexto social e histórico vigente.

Se requiere adoptar una metodología dinámica y paciente que incluya no solamente el desarrollo de competencias, el uso de TIC, sino también fases prácticas que involucre salir del campus y lo lleve al logro de las metas "Esto plantea la necesidad de diversificar las modalidades de trabajo en el aula a través de talleres, seminarios, laboratorios y aún más, a través de la posibilidad de promover la capacidad en el alumno de transferir el conocimiento del mundo del saber al mundo cotidiano, la ciencia al mundo del trabajo profesional, y de establecer una relación compleja entre el saber y el trabajo en la esfera del conocimiento de alto nivel y en el de las tareas cognoscitivas complejas.

Fuente. Mazuelos (2022) 


\section{CONCLUSIONES}

Esta investigación permitió determinar, desde la perspectiva de los estudiantes y profesores de la facultad de educación de la universidad en la que se realizó el estudio, que

1. Las competencias afectivas del docente son de gran importancia para el aprendizaje. Estas pueden impactar positivamente y colaborar en el logro de los objetivos formativos que se hayan trazado. Por ello, se espera un docente que sea jovial, auténtico, empático, abierto, comprometido, flexible y comprensivo.

2. Las competencias sociales, aquellas que permiten que el docente relaciones cercanas y positivas con su estudiante, son de vital importancia. Ya que este tipo de relaciones positivas permiten al docente conocer mejor las peculiaridades y necesidades de los estudiantes, prevenir situaciones y solucionar sus problemas para conseguir su bienestar integral.

3. Las competencias comunicativas, son sub-competencias personales que están implicadas en las competencias sociales. Estas competencias comunicativas están referidas tanto a los lenguajes formales (académico, investigativo, etc.), como a las habilidades expresivas físicas y verbales (coherencia y asertividad) y a la actitud de escucha activa. La relevancia de este grupo de competencias comunicativas radica en que son el instrumento que permite lograr relaciones positivas con el estudiante, las que potencialmente llevan al estudiante al logro de sus metas de formación.

4. Las competencias blandas o personales referidas a rasgos de personalidad (conclusiones 1-3), de socialización y comunicación tienen un papel de gran importancia en la formación integral del estudiante.

5. Las competencias de orientación al logro están consideradas dentro de las competencias laborales del docente de educación de la facultad y no dentro de las competencias personales puesto que deben estar encaminadas a formar un profesional competente y un ser humano reflexivo.

6. La competencia laboral de gestión de la información está circunscrita dentro del uso de las tecnologías de la información y la comunicación con propósitos de enseñanzaaprendizaje. El docente debe seguir superarando la brecha en el uso de TIC a través del dominio de su didáctica, lo que a la vez le facilitará la innovación en este campo y mantener la motivación de sus estudiantes. 
7. El docente de la facultad en la que se realizó el estudio debe conocer los aspectos teóricos y prácticos de su área, conocimientos sobre su realidad, con a tención a la diversidad; debe saber de pedagogía y de investigación que le permita adecuar los contenidos a la realidad en la que ejerce (competencias disciplinares o cognitivas).

8. Las competencias meta-cognitivas son de gran importancia para determinar metas del qué y cómo enseñar/aprender; para estar auto motivados, y tener compromiso para la autoevaluación para la mejora.

9. Las competencias didácticas, están más orientadas hacia las competencias metodológicas. Estas implican que además de tener las metas de enseñanzaaprendizaje, de planificación y evaluación claras, se requiere de una metodología dinámica y paciente, centrada en el estudiante, que incluyan el desarrollo de competencias, el uso de TIC, también fases prácticas fuera del campus y lo lleve al logro de las metas, todo esto considerando siempre, las expectativas del estudiante.

10. De las conclusiones 1,2 y 3 referidas a competencias personales, y de las conclusiones $5,6,7,8$ y 9 , referidas a competencias laborales, se puede inferir que los estudiantes y profesores entrevistados estiman que las competencias profesionales del docente de la facultad de educación en la que se realizó el estudio de caso, están conformadas por 1) competencias personales, que a la vez están formadas por las sub-competencias:

a) Competencias afectivas (personalidad del profesor); b) competencias sociales y c) competencias comunicativas de trascendencia en la formación de relaciones cercanas para un mejor aprendizaje. Y 2) competencias laborales, conformadas por las competencias: a) competencias de orientación al logro; b) competencias para la gestión de la información, orientadas hacia las competencias para el uso de las tecnologías de la información y la comunicación con propósitos de enseñanzaaprendizaje; c) competencias disciplinares o cognitivas, dentro de las que se encuentran las competencias investigativas; d) competencias meta-cognitivas y e) competencias didácticas orientadas hacia las competencias metodológicas.

\section{LISTA DE REFERENCIAS}

Aguirre, A (2005). Reflexiones acerca de la competencia comunicativa profesional. Educ Med Super v.19 n.3 Ciudad de la Habana jul.-sep. 2005. Recuperado de http://scielo.sld.cu/scielo.php?script=sci_arttext\&pid=S086421412005000300004 
Barrón, M. (Ene. 2009). Docencia universitaria y competencias didácticas. Revista Perfiles educativos. vol.31 no.125. México. Recuperado de http://www.scielo.org.mx/scielo.php?script=sci_arttext\&pid=S018526982009000300006

Bisquerra, R. (s.f.). Competencia social. Grop de Recerca de Orientació PsicopedagógicaUniversit de Barcelona. http://www.rafaelbisquerra.com/es/competenciasemocionales/competencia-social.html

Bolseguí, M. \& Fuguet, A. (2006). Construcción de un modelo conceptual a través de la investigación cualitativa Sapiens. Revista Universitaria de Investigación, vol. 7, núm. 1, junio, pp. 207-229 Universidad Pedagógica Experimental Libertador Caracas, Venezuela

Boyatzis, R. (1982). The competent manager. Argentina-New York: Wiley and Sons.

Correa, G. Capacidad de emprendimiento del joven universitario. Estudio de caso. Gestión \& Sociedad, [S.1.], v. 2, n. 2, p. 75-88, dec. 2009. ISSN 2027-1433. Disponible en: <http://revistas.lasalle.edu.co/index.php/gs/article/view/1121>. Fecha de acceso: 29 feb. 2016. Recuperado de: http://www3.weforum.org/docs/WEF_GlobalCompetitivenessReport_201415.pdf

Demuth, P. (2011). Conocimiento profesional docente: conocimiento académico, saber experiencial, rutinas y saber tácito. Revista del Instituto de Investigaciones en Educación. Facultad De Humanidades - UNNE.

Dolan, S., Valle, R., Jackson, S. \& Schuler, R. (2007). La gestión de los recursos humanos: cómo atraer, retener y desarrollar con éxito el capital humano en tiempos de transformación. España: McGraw-Hill (3a. ed.).

Donaldson, G. (2012). Development of National Teacher Qualification Frameworks Across Five Balkan Countries. University of Glasgow. Recuperado de http://www.cep.edu.rs/sites/default/files/teacher_qualifications_in_five_balkan_ countries.pdf

Estrada, S. (S,f.). Orientación al Logro. Universidad tecnológica de Pereira. Recuperado de: http://univirtual.utp.edu.co/pandora/recursos/0/791/791.pdf 
Eunjung, L., Mishna, F. \& Brennenstuhl, S. (2010). How to critically evaluate case studies in social work. Research on Social Work Practice, 20(6), November 2010, pp.682689.

García, M.B. (2011). [proyecto] “Análisis de experiencias de enseñanza aprendizaje de la competencia comunicativa escrita a nivel de Ingeniería Informática". Universidat Oberta de Catalunya (UOC).

Galvis, R. (2007). De un perfil docente tradicional a un perfil docente basado en competencias. Acción pedagógica, No 16 / Enero - Diciembre, 2007 - pp. 48 - 57. Recuperado de file:///C:/Users/Sandra/Downloads/DialnetDeUnPerfilDocenteTradicionalAUnPerfilDocenteBasado-2968589.pdf

Garrido, C. (2007). La educación desde la teoría del capital humano y el otro. pp. 73-80. Educere Vol.11 (36, supl.36) Meridad. Recuperado de http://www.scielo.org.ve/scielo.php?pid=S131649102007000100010\&script=sci_arttext

Junta de Castilla y León (s.f). Red de formación del Profesorado "Competencia didáctica). Recuperado de http://csfp.centros.educa.jcyl.es/sitio/upload/didactica_def.pdf

Korthagen, F. (2003). In search of the essence of a good teacher: towards a more holistic approach in teacher education. Teaching and Teacher Education 20 (2004) 77-97. IVLOS Institute of Education, Utrecht University. Recuperado de http://cmapsconverted.ihmc.us/rid=1181134774565_1500279159_13957/essenc e\%20of\%20a\%20good\%20teacher.pdf

López Arce, A. (2006). El currículo en la educación superior: un enfoque posmoderno basado en competencias. Recuperado de https://books.google.com.pe/books?isbn=9682002567 - Translate this page

Marinković, S., Bjekić, D. \& Zlatić,L. (2012). Teachers' Competence as the Indicator of the Quality and Condition of Education1. University of Kragujevac, Serbia. Recuperado de http://www.tepe2012.uni.lodz.pl/uploads/ThemenIV/Marinkovi\%C4\%87,\%20Sn ezana;\%20Bjeki\%C4\%87,\%20Dragana\%20\&\%20Zlati\%C4\%87,\%20Lidija.pdf

McKinsey \& Company (2007). How the world's best performing school systems come out on top. Recuperado de http://mckinseyonsociety.com/how-the-worlds-bestperforming-schools-come-out-on-top/ 
Orama, R., Tamine, J. Y Varcárcel, N. (2013). Educación Médica Superior, vol.27 no.1. Ciudad de la Habana. Versión ISSN 0864-2141. Recuperado de: http://scielo.sld.cu/scielo.php?pid=S0864-

$21412013000100015 \&$ script $=$ sci_arttext $\&$ tlng $=$ pt

Parra, E. (2005). Formación por competencias: una decisión para tomar dentro de posturas encontradas. Revista Virtual Universidad Católica del Norte. Recuperado de: http://www.redalyc.org/pdf/1942/194220418015.pdf

Pavié, A. (2012). Las competencias profesionales del profesorado de lengua castellana y comunicaciones en Chile: Aportaciones a la formación inicial. (Tesis doctoral). Universidad de Valladolid. Recuperado de http://uvadoc.uva.es/bitstream/10324/2794/1/TESIS297-130508.pdf

Perrenoud, P. (2001). La formación del docente del siglo XXI. Montevideo: Cinterfor.

Rivera, G. (2006). Modelo de competencias profesionales para docentes de educación básica según el criterio de un grupo de educadores. (Tesis para obtener el grado de Doctor en filosofía con especialidad en educación). Recuperado de http://cdigital.dgb.uanl.mx/te/1020160583/1020160583_MA.PDF

Rodríguez, G., Gil, J. \& García, E. (1996). Metodología de la investigación cualitativa. Granada: Ediciones Aljibe

Rodríguez, N. (2011) Estrategias didácticas para el desarrollo de competencias básicas del profesional universitario peruano. Recuperado de http://sisbib.unmsm.edu.pe/bibvirtual/publicaciones/inv_educativa/2011_n28/pd f/a02v15n28.pdf

Ruíz, J. (2003). Metodología de la investigación cualitativa $4^{\circ}$ edición. Capítulo 1: la investigación cualitativa. Cap-1-1-y-2. ISBN : 978-84-7485-423-7: Universidad de Deusto

Sandín, M.P. (2000). Criterios de validez en la investigación cualitativa: de la objetividad a la solidaridad. Revista de Investigación Educativa, Vol. 18, n. ${ }^{\circ}$ 1, págs. 223-242. Recuperado de file://C:/Users/Sandra/Downloads/121561-481061-1PB\%20(1).pdf

Spencer, L. \& Spencer, S. (1993) “Competence at Work”. Jhon Wiley \& Sons, Toronto, 1993. Orientación al logro Recuperado de: http://univirtual.utp.edu.co/pandora/recursos/0/791/791.pdf 
Su-Chin Hsieh, Jui-Shin Lin, y Hung-Chun Lee. (2012). Analysis on Literature Review of Competency. International Review of Business and Economics. Vol.2, pp.2550. Unpublished doctoral dissertation, George Washington University, Washington, DC. October 2012.

Tejada, J. (2009). Competencias Docentes. Teachers Competencies. Vol. 13 (2). ISSN 1138-414X. Recuperado de https://www.ugr.es/ recfpro/rev132COL2.pdf

Ugbe, A. \& Agim, J. (2009). Influence of teachers' competence on student academic performance in senior secondary school chemistry Global Journal of Educational Research. Vol 8 (1-2). 